\title{
Apoptosis and inhibition of proliferation of cancer cells induced by cordycepin (Review)
}

\author{
XUEWEN TIAN ${ }^{1,2}$, YUJIAN LI ${ }^{3}$, YINYU SHEN ${ }^{3}$, QIAOQIAO LI $^{3}$, QINGLU WANG ${ }^{3}$ and LIANSHI FENG ${ }^{1,4}$ \\ ${ }^{1}$ School of Kinesiology, Shanghai University of Sport, Shanghai 200438; ${ }^{2}$ Sports Science Research Center of Shandong, \\ Jinan, Shandong 250102; ${ }^{3}$ Key Laboratory of Biomedical Engineering and Technology of Shandong High School, \\ Shandong Wanjie Medical College, Zibo, Shandong 255213; ${ }^{4}$ Biology Center, \\ China Institute of Sport Science, Beijing 100061, P.R. China
}

Received June 12, 2014; Accepted February 24, 2015

DOI: $10.3892 / \mathrm{ol} .2015 .3273$

\begin{abstract}
Cordycepin, a 3-deoxyadenosine, is the predominant functional component of the fungus Cordyceps militaris, a traditional Chinese medicine. Previous studies investigating the inhibition of cancer cells by cordycepin identified that it not only promotes cell apoptosis, but also controls cell proliferation. Furthermore, studies have elucidated the molecular mechanisms of inhibiting cell proliferation by cordycepin binding the A3 adenosine receptor, activating $G$ protein, inhibiting cAMP formation, decreasing glycogen synthase kinase- $3 \beta / \beta$-catenin activation and suppressing cyclin D1 and c-myc expression. The most significant signaling pathway in which cell apoptosis is induced by cordycepin is the caspase pathway. Cordycepin induces cell apoptosis via binding the DR3 receptor and consequently activating caspase-8/-3. Taken together, these studies demonstrate that cordycepin may be used as a natural medicine, as it can not only control tumor cell proliferation, but also induce cancer cell apoptosis.
\end{abstract}

\section{Contents}

1. Introduction

2. Cell proliferation inhibition induced by cordycepin

3. Cell apoptosis induced by cordycepin

4. Conclusions and perspectives

Correspondence to: Dr Qinglu Wang, Key Laboratory of Biomedical Engineering and Technology of Shandong High School, Shandong Wanjie Medical College, 246 Wucheng Road, Zibo, Shandong 255213, P.R. China

E-mail: wqlzcq@gmail.com

Professor Lianshi Feng, School of Kinesiology, Shanghai University of Sport, 650 Qingyuan Ring Road, Shanghai 200438, P.R. China E-mail: fengls98@126.com

Key words: cordycepin, cancer cells apoptosis, cell proliferation, caspase signal pathway

\section{Introduction}

Cancer is caused by an imbalance between cell cycle progression and apoptosis. Therefore, the majority of anticancer drugs exert their antiproliferative and cytotoxic activity via cell cycle arrest and induction of apoptosis, a controlled form of cell death that is dysregulated in cancer (1). Cytotoxic nucleoside analogues were the first chemotherapeutic agents used for the treatment of cancer (2). To date, the most widely researched cytotoxic nucleoside analogues are predominantly isolated from Cordyceps sinensis and C. militaris.

$C$. sinensis has been more extensively used and investigated compared with $C$. militaris, however, both species contain similar bioactive ingredients and exhibit medicinal activity, and are widely used traditional Chinese medicines. A number of bioactive ingredients have been isolated from C. militaris, including adenosine, cordycepin, D-mannitol, and exopolysaccharides. $C$. militaris has been widely used in traditional Chinese medicine (3), and is claimed to have extensive pharmacological properties, including anti-inflammatory properties, cell cycle disruption, enhancement of immune function, nucleic acid-containing (DNA/RNA) and apoptosis-inducing activities; anti-fatigue, anti-bacterial and anti-diabetic properties; improving lung, liver and kidney functions; and aiding in the treatment of cancer as well as male and female sexual dysfunctions (3). C. militaris is therefore receiving much attention due to these potential health benefits, and it is often used as a substitute for $C$. sinensis in certain traditional Chinese medicine prescriptions as the two species contain similar bioactive ingredients (4). In view of the growing popularity of C. militaris, cultivation of the $C$. militaris stroma, a spore of C. militaris, is also conducted. A wide diversity of compounds have been isolated from different strains of Cordyceps as well as artificially cultivated $C$. militaris (4).

Cordycepin, also known as 3-deoxyadenosine, is a specific polyadenylation inhibitor and is the main functional component in C. militaris (5). Cordycepin is an active small molecule and is implicated in modulating multiple physiological functions, including immune-activation (anti-virus, anti-infection) (6), anti-inflammatory, anti-aging (adjustment of the physical condition, an anticancer effect and enhancement of sexual performance) (7) and anti-tumor effects (8). 


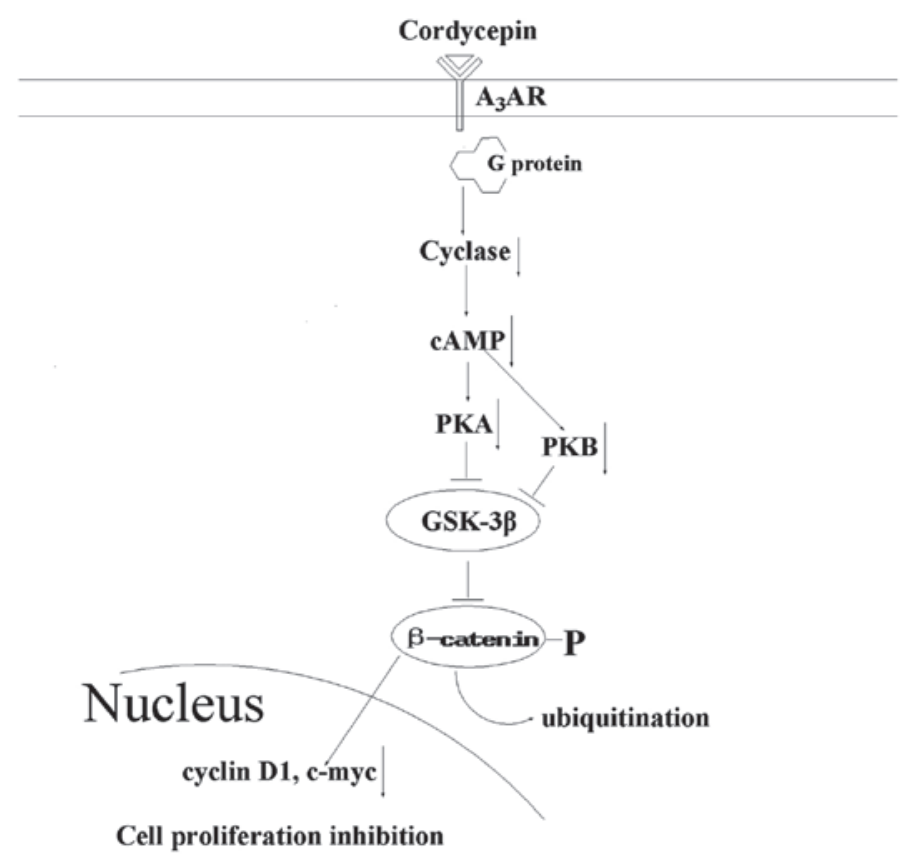

Figure 1. Signaling pathway of cell proliferation inhibition under cordycepin stress.

Throughout the last 60 years, the antitumor mechanism of Cordycepin has become extensively studied. In 1961, Jagger et al (9) investigated the inhibition of Ehrlich mouse ascites tumor growth by cordycepin and Klenow (10) also investigated the effect of cordycepin on the incorporation of P32-orthophosphate into the nucleic acid of ascites tumor cells in vitro. These studies prompted further research into the association between cordycepin and cancer. In the subsequent decades, numerous studies with regard to cordycepin and cancer were reported. For example, cordycepin was shown to induce antitumor effects or apoptosis in human head and neck squamous cell carcinoma (11), bladder cancer (12), thyroid carcinoma (13), breast cancer (14), multiple myeloma (15), leukemia $(5,16,17)$, lymphoma (18) and mouse leydig tumor cells (19). Additionally, the inhibitory effect of cordycepin was observed in hematogenic metastasis of mouse melanoma $(20,21)$ and lung carcinoma cells $(22)$. Cell cycle arrest was observed to be promoted by cordycepin via the regulation of c-Jun N-terminal kinase (JNK) in human bladder and colon cancer cells $(23,24)$. In hematological malignancies, cordycepin exhibited cytotoxic and apoptogenic effects via the inactivation of polyadenylate polymerase and the resulting inhibition of mRNA polyadenylation (18). In terminal deoxynucleotidyl transferase-positive leukemic cells, these effects are more prominent (18).

\section{Cell proliferation inhibition induced by cordycepin}

It is important to understand how cordycepin inhibits cell proliferation in tumor cells in order to develop it as a new agent for the prevention and treatment of cancer. The $\mathrm{A} 3$ adenosine receptor $\left(\mathrm{A}_{3} \mathrm{AR}\right)$ is a member of the AR family; it is overexpressed in cancer and inflammatory cells, however, low expression is exhibited in normal cells (25). Following cordycepin binding to $\mathrm{A}_{3} \mathrm{AR}, \mathrm{G}$ protein is activated and subsequently inhibits the formation of cAMP and indirectly decreases phosphorylation of the serine/threonine kinase glycogen synthase kinase (GSK)-3 $\beta$. The resulting increased phosphorylation of $\beta$-catenin causes it to be removed from the cytoplasm by ubiquitination, thereby preventing its nuclear import. A net suppression of cyclin D1 and c-myc is the result of this, which leads to the inhibition of cell growth (Fig. 1) (26). Thus, as cordycepin binds $A_{3} A R$, which inactivates the GSK-3 $\beta / \beta$-catenin signaling pathway and subsequently suppresses cell division, cordycepin may present a potential therapeutic agent for the inhibition of tumor cell proliferation.

\section{Cell apoptosis induced by cordycepin}

Protein kinase A (PKA)/terminal deoxynucleotidyl transferase (TdT) signaling pathway. In 1996, Koc et al (27) speculated that cordycepin monophosphate in TdT-positive cells may be able to activate PKA in place of cAMP, and that PKA may phosphorylate TdT, augmenting its activity as an endonuclease. In cell-free experiments, the activity of recombinant TdT as an endonuclease digesting supercoiled plasmid DNA into linear fragments was significantly increased following phosphorylation of TdT by PKA. Therefore, the potential role of $\mathrm{TdT}$ as an apoptotic endonuclease in TdT-positive leukemia cells following cordycepin exposure requires investigation in the future.

Caspase signaling pathway. Cordycepin is also involved in apoptosis through the caspase pathway. In 2011, Jen et al (19) confirmed that cordycepin was able to induce MA-10 mouse Leydig tumor cell apoptosis via the caspase-9 pathway (Fig. 2), where cordycepin administration was found to increase the expression of caspase- $9,-3$ and -7 proteins. The same year, Jeong et al (5) proposed a mechanism by which cordycepin induces the apoptosis of human leukemia cells through a signaling cascade involving a reactive oxygen species-mediated caspase pathway, which was indicated by the 


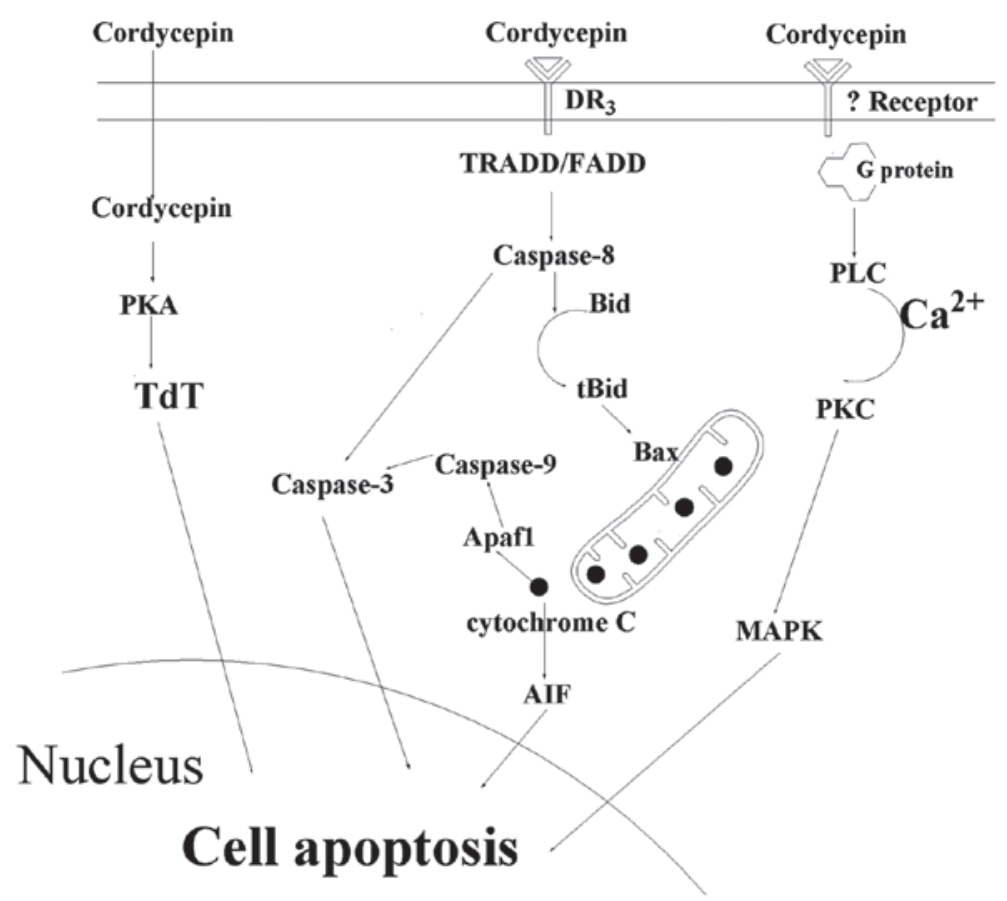

Figure 2. Signaling pathway of cell apoptosis under cordycepin stress.

generation of reactive oxygen species, mitochondrial dysfunction, activation of capsases and cleavage of poly (ADP ribose) polymerase 1 (PARP) in the study. Imesch et al (28) demonstrated activated caspase-dependent, intrinsic apoptosis, which was indicated by the proteolytic cleavage of caspase-9, caspase-3 and PARP. Choi et al (29) showed that cordycepin-induced cell death in MDA-MB-231 cells was associated with the translocation of Bax from the cytosol to the mitochondria, a feature of the mitochondria-mediated apoptotic pathway; this was confirmed by DNA fragmentation, terminal deoxynucleotidyl transferase dUTP nick end labeling, and immunocytochemical analysis. Additionally, cordycepin induced a dose-dependent increase in mitochondrial translocation of Bax, causing the cytosolic release of cytochrome $c$ and activation of caspase-9 and -3. Notably, autophagy-associated cell death was observed for MCF-7 cells, demonstrated by the detection of an autophagosome-specific protein and large membranous vacuole ultrastructure morphology in the cytoplasm.

Cell proliferation is inhibited by cordycepin via binding to $\mathrm{A}_{3} \mathrm{AR}$; however programmed cell death will occur following the interaction of cordycepin and the death receptor, DR3, which is a death-domain-containing tumor necrosis factor family receptor (30). A previous study demonstrated that the expression levels of certain proteins related to apoptosis, including p53 and Bax, were increased following treatment with cordycepin for $18 \mathrm{~h}$, and also confirmed that DR3, caspase-8, caspase-1, cleaved caspase-3 and cleaved PARP expression was increased (31). After cordycepin binding to the DR3 receptor, DR3 recruits initiator caspase- 8 via the adaptor protein tumor necrosis factor receptor type 1-associated DEATH domain protein/Fas-associated protein with death domain (FADD). Caspase- 8 subsequently oligomerizes, and is activated via autocatalysis. This indicates that activated caspase- 8 stimulates apoptosis via two parallel cascades: Direct activation of caspase-3 or the release of cytochrome $c$.
Direct activation of caspase-3. Caspase- 8 may directly cleave and activate caspase-3 (Fig. 2). In particular, caspase-3 (also known as CPP32/Yama/apopain) (32-34) comprises a $32 \mathrm{kDa}$ zymogen that is cleaved into $17 \mathrm{kDa}$ and $12 \mathrm{kDa}$ subunits when it interacts with caspase-8/9. When the procaspase is cleaved at a particular residue, the active heterotetramer may then form via hydrophobic interactions, resulting in four anti-parallel $\beta$-sheets from p17 and two from p12 to combine to form a heterodimer. The zymogen feature of caspase-3 is necessary as, if unregulated, caspase activity would kill cells indiscriminately. Caspase 3 is an executioner caspase, and therefore its zymogen has almost no activity until it is cleaved by an initiator caspase after apoptotic signaling events have occurred (35). Western blot analysis demonstrated the induction of active caspase- 3 and PARP cleavage by cordycepin treatment, indicating that cordycepin is able to activate the caspase-3 pathway (36).

Release of cytochrome $c$. When caspase-3 is activated, concurrently, caspase- 8 also cleaves the pro-apoptotic Bcl- 2 family protein, Bid. Following this, truncated Bid (tBid) translocates to the mitochondria and induces cytochrome $c$ release, which results in an increase of the Bax/Bcl-xL ratio (37). Cytochrome $c$ sequentially binds apoptotic protease activating factor-1 (Apaf1), forms an activation complex with caspase-9 and activates caspase-3 (3). Furthermore, cytochrome $c$ has been demonstrated to activate apoptosis-inducing factor, which migrates to the nucleus and induces cell apoptosis; however, the associated signaling pathway is yet to be confirmed experimentally (3).

p38/JNK signaling pathway. Based on the increased expression of PKC, extracellular signal-regulated kinase $1 / 2$ (ERK1/2) and c-JNK, as determined by Western blot analysis, Pao et al (7) reported that cordycepin stimulated intracellular phospholipase $\mathrm{C} / \mathrm{PKC}$ and mitogen-activated protein kinase 
(MAPK) signal transduction pathways to induce cell death in MA-10 mouse Leydig tumor cells. This result indicated that cordycepin induces tumor cell death through PKC/MAPK signaling pathways. Lee et al (38) performed Western blot analysis, which demonstrated that JNK-inactivating phosphatase was upregulated in response to treatment with cordycepin in human hepatocellular carcinoma Hep3B cells. Thus, according to the results of Pao et al (7) and Lee et al (38), we hypothesize that cordycepin induces cell apoptosis via the activation of PLC/PKC and subsequent inactivation of JNK, which is an important component of the MAPK and downstream PLC/PKC signaling pathways. Western blot analysis performed by Chen et al $(39,40)$ also found that $24 \mathrm{~h}$ treatment with 10 or $100 \mu \mathrm{mol} / 1$ cordycepin exhibited a synergistically apoptotic effect through the activation of JNK/caspase-7/PARP pathway in human OC3 oral cancer cells. These analyses indicate that cordycepin may also induce cancer cell apoptosis/death by PKC, MAPK, JNK, caspase-7, and PARP pathways.

\section{Conclusions and perspectives}

Collectively, the findings that are discussed in the present review provide a novel insight into the effect of cordycepin on cell apoptosis, which supports new concepts for the treatment of cancer. Cell proliferation and apoptosis involve the functional cooperation of many signaling molecules. As summarized in Fig. 1, cordycepin inhibits cell proliferation via binding $\mathrm{A}_{3} \mathrm{AR}$, activating $\mathrm{G}$ protein, inhibiting cAMP formation, decreasing GSK-3 $\beta / \beta$-catenin activation and suppressing cyclin D1 and c-myc expression.

Cordycepin induces cell apoptosis through three signaling pathways: PKA/TdT signaling pathway, caspase signaling pathway and $\mathrm{p} 38 / \mathrm{JNK}$ signaling pathway. The caspase signaling pathway is the most prominent signal pathway by which cell apoptosis is induced by cordycepin. Following cordycepin binding to the DR3 receptor, DR3 activates caspase- 8 via FADD. Subsequently, caspase- 8 may directly activate caspase- 3 or induce mitochondria to release cytochrome $c$, which also activates caspase-3 with the help of Apaf1 and caspase-9.

These results indicate that a cocktail therapy with cordycepin may greatly reduce the risk of cancer cell metastasis of all types. The significance of cordycepin as a natural medicine is that may be used to treat or prevent cancer progression in the future (41). Further studies are required to determine which of the signaling pathways is most important for the treatment of cancer with cordycepin. In addition, many of the previous studies were focused on the activity of cordycepin at a cellular level. Future in vivo studies investigating the inhibition of tumor progression by cordycepin may provide further insight into the mechanisms behind its activity.

\section{Acknowledgements}

This work was supported by grants from the Natural Scientific Foundation of Chinese Shandong Province (grant no. ZR2010CQ031 and ZR2014CM046) and Shanghai Key Lab of Human Performance, Shanghai University of Sport (grant no. 11DZ2261100).

\section{References}

1. Thomadaki H, Tsiapalis CM and Scorilas A: Polyadenylate polymerase modulations in human epithelioid cervix and breast cancer cell lines, treated with etoposide or cordycepin, follow cell cycle rather than apoptosis induction. Biol Chem 386: 471-480, 2005.

2. Tuli HS, Sharma AK, Sandhu SS and Kashyap D: Cordycepin: A bioactive metabolite with therapeutic potential. Life Sci 93: 863-869, 2013.

3. Lee HH, Park C, Jeong JW, et al: Apoptosis induction of human prostate carcinoma cells by cordycepin through reactive oxygen species-mediated mitochondrial death pathway. Int J Oncol 42: 1036-1044, 2013.

4. Lim L, Lee C and Chang E: Optimization of solid state culture conditions for the production of adenosine, cordycepin and D-mannitol in fruiting bodies of medicinal caterpillar fungus cordyceps militaris (L:Fr.) Link (Ascomycetes). Int J Med Mushrooms 14: 181-187, 2012.

5. Jeong JW, Jin CY, Park C, et al: Induction of apoptosis by cordycepin via reactive oxygen species generation in human leukemia cells. Toxicol In vitro 25: 817-824, 2011.

6. Kim HG, Shrestha B, Lim SY, et al: Cordycepin inhibits lipopolysaccharide-induced inflammation by the suppression of NF-kappaB through Akt and p38 inhibition in RAW 264.7 macrophage cells. Eur J Pharmacol 545: 192-199, 2006.

7. Pao HY, Pan BS, Leu SF and Huang BM: Cordycepin stimulated steroidogenesis in MA-10 mouse leydig tumor cells through the protein kinase C pathway. J Agric Food Chem 60: 4905-4913, 2012

8. Yao WL, Ko BS, Liu TA, et al: Cordycepin suppresses integrin/FAK signaling and epithelial-mesenchymal transition in hepatocellular carcinoma. Anticancer Agents Med Chem 14: 29-34, 2014.

9. Jagger Dv, Kredich Nm and Guarino AJ: Inhibition of Ehrlich mouse ascites tumor growth by cordycepin. Cancer Res 21: 216-220, 1961.

10. Klenow, H: Effect of cordycepin on the incorporation of P32-orthophosphate into the nucleic acids of ascites tumor cells in vitro. Biochem Biophys Res Commun 5: 156-9, 1961.

11. Wu WC, Hsiao JR, Lian YY, Lin CY and Huang BM: The apoptotic effect of cordycepin on human OEC-M1 oral cancer cell line. Cancer Chemother Pharmacol 60: 103-111, 2007.

12. Lee EJ, Kim WJ and Moon SK: Cordycepin suppresses TNF-alpha-induced invasion, migration and matrix metalloproteinase-9 expression in human bladder cancer cells. Phytother Res 24: 1755-1761, 2010.

13. Chen Y, Chen YC, Lin YT, Huang SH and Wang SM: Cordycepin induces apoptosis of CGTH W-2 thyroid carcinoma cells through the calcium-calpain-caspase 7-PARP pathway. J Agric Food Chem 58: 11645-11652, 2010.

14. Lee HJ, Burger P, Vogel M, et al: The nucleoside antagonist cordycepin causes DNA double strand breaks in breast cancer cells. Invest New Drugs 30: 1917-1925, 2012.

15. Chen LS, Stellrecht CM and Gandhi V: RNA-directed agent, cordycepin, induces cell death in multiple myeloma cells. Br J Haematol 140: 682-391, 2008.

16. Matsuda H, Akaki J, Nakamura S, et al: Apoptosis-inducing effects of sterols from the dried powder of cultured mycelium of Cordyceps sinensis. Chem Pharm Bull (Tokyo) 57: 411-414, 2009.

17. Kodama EN, McCaffrey RP, Yusa K and Mitsuya H: Antileukemic activity and mechanism of action of cordycepin against terminal deoxynucleotidyl transferase-positive (TdT+) leukemic cells. Biochem Pharmacol 59: 273-281, 2000.

18. Thomadaki H, Tsiapalis CM and Scorilas A: The effect of the polyadenylation inhibitor cordycepin on human Molt-4 and Daudi leukaemia and lymphoma cell lines. Cancer Chemother Pharmacol 61: 703-711, 2008.

19. Jen CY,Lin CY,Huang BM and Leu SF: Cordycepin induced MA-10 mouse leydig tumor cell apoptosis through Caspase-9 pathway. Evid Based Complement Alternat Med 2011: 984537, 2011.

20. Yoshikawa N, Kunitomo M, Kagota S, Shinozuka K and Nakamura K: Inhibitory effect of cordycepin on hematogenic metastasis of B16-F1 mouse melanoma cells accelerated by adenosine-5'-diphosphate. Anticancer Res 29: 3857-3860, 2009.

21. Yoshikawa N, Yamada S, Takeuchi C, et al: Cordycepin (3'-deoxyadenosine) inhibits the growth of B16-BL6 mouse melanoma cells through the stimulation of adenosine $\mathrm{A} 3$ receptor followed by glycogen synthase kinase-3beta activation and cyclin D1 suppression. Naunyn Schmiedebergs Arch Pharmacol 377: 591-595, 2008. 
22. Nakamura K, Yoshikawa N, Yamaguchi Y, et al: Antitumor effect of cordycepin (3'-deoxyadenosine) on mouse melanoma and lung carcinoma cells involves adenosine A3 receptor stimulation. Anticancer Res 26: 43-47, 2006.

23. Lee SJ, Kim SK, Choi WS, Kim WJ and Moon SK: Cordycepin causes p21WAF1-mediated G2/M cell-cycle arrest by regulating c-Jun N-terminal kinase activation in human bladder cancer cells. Arch Biochem Biophys 490: 103-109, 2009.

24. Lee SJ, Moon GS, Jung KH, Kim WJ and Moon SK: c-Jun $\mathrm{N}$-terminal kinase 1 is required for cordycepin-mediated induction of G2/M cell-cycle arrest via p21WAF1 expression in human colon cancer cells. Food Chem Toxicol 48: 277-283, 2010.

25. Madi L, Ochaion A, Rath-Wolfson L, et al: The A3 adenosine receptor is highly expressed in tumor versus normal cells: potential target for tumor growth inhibition. Clin Cancer Res 10: 4472-4479, 2004

26. Fishman P, Bar-Yehuda S, Liang BT and Jacobson KA Pharmacological and therapeutic effects of A3 adenosine receptor agonists. Drug Discov Today 17: 359-366, 2012.

27. Koc Y, Urbano AG, Sweeney EB and McCaffrey R: Induction of apoptosis by cordycepin in ADA-inhibited TdT-positive leukemia cells. Leukemia 10: 1019-1024, 1996.

28. Imesch P, Hornung R, Fink D and Fedier A: Cordycepin (3'-deoxyadenosine), an inhibitor of mRNA polyadenylation, suppresses proliferation and activates apoptosis in human epithelial endometriotic cells in vitro. Gynecol Obstet Invest 72: 43-49, 2011.

29. Choi S, Lim MH, Kim KM, Jeon BH, Song WO and Kim TW: Cordycepin-induced apoptosis and autophagy in breast cancer cells are independent of the estrogen receptor. Toxicol Appl Pharmacol 257: 165-173, 2011.

30. Chinnaiyan AM, O'Rourke K, Yu GL, Lyons RH, Garg M, Duan DR, Xing L, Gentz R, Ni J and Dixit VM: Signal transduction by DR3, a death domain-containing receptor related to TNFR-1 and CD95. Science 274: 990-992, 1996.

31. Lee SY, Debnath T, Kim SK and Lim BO: Anti-cancer effect and apoptosis induction of cordycepin through DR3 pathway in the human colonic cancer cell HT-29. Food Chem Toxicol 60: 439-447, 2013.
32. Nicholson DW, Ali A, Thornberry NA, et al: Identification and inhibition of the ICE/CED-3 protease necessary for mammalian apoptosis. Nature 376: 37-43, 1995.

33. Tewari M, Quan LT, O'Rourke K, et al: Yama/CPP32 beta, a mammalian homolog of CED-3, is a CrmA-inhibitable protease that cleaves the death substrate poly (ADP-ribose) polymerase. Cell 81: 801-809, 1995.

34. Fernandes-Alnemri T, Litwack G and Alnemri ES: CPP32, novel human apoptotic protein with homology to Caenorhabditis elegans cell death protein Ced-3 and mammalian interleukin-1 beta-converting enzyme. J Biol Chem 269: 30761-30764, 1994.

35. Walters J, Pop C, Scott FL, et al: A constitutively active and uninhibitable caspase-3 zymogen efficiently induces apoptosis. Biochem J 424: 335-345, 2009.

36. Baik JS, Kwon HY, Kim KS, Jeong YK, Cho YS and Lee YC: Cordycepin induces apoptosis in human neuroblastoma SK-N-BE (2)-C and melanoma SK-MEL-2 cells. Indian J Biochem Biophys 49: 86-91, 2012.

37. Shimizu S and Tsujimoto Y: Proapoptotic BH3-only Bcl-2 family members induce cytochrome c release, but not mitochondrial membrane potential loss, and do not directly modulate voltage-dependent anion channel activity. Proc Natl Acad Sci USA 97: 577-582, 2000.

38. Lee HH, Jeong JW, Lee JH, et al: Cordycepin increases sensitivity of Hep3B human hepatocellular carcinoma cells to TRAIL-mediated apoptosis by inactivating the JNK signaling pathway. Oncol Rep 30: 1257-1264, 2013.

39. Chen YH, Hao LJ, Hung CP, Chen JW, Leu SF and Huang BM: Apoptotic effect of cisplatin and cordycepin on OC3 human oral cancer cells. Chin J Integr Med 20: 624-632, 2014

40. Chen YH, Wang JY, Pan BS, et al: Cordycepin enhances cisplatin apoptotic effect through caspase/MAPK pathways in human head and neck tumor cells. Onco Targets Ther 6: 983-998, 2013.

41. Tian X, Zhao X, Yin K, Mao D, Yang J and Wang Q: Immunoregulation ability comparison of cordycepin and Flammulina velutipes polysaccharide in rat body after exhaustive exercise. Int J Biol Biol Sci 2: 136-142, 2013. 